05

\title{
Использование планарных пермаллоевых микрочастиц для детектирования механических напряжений
}

\author{
() Н.И. Нургазизов, ${ }^{1}$ Д.А. Бизяев, ${ }^{1}$ А.А. Бухараев, ${ }^{1}$ И.В. Русских, ${ }^{1}$ Ю.В. Садчиков ${ }^{1,2}$ \\ ${ }^{1}$ Казанский фризико-технический институт им. Е.К. Завойского, \\ Федеральный исследовательский центр „Казанский научный центр РАН“, \\ 420029 Казань, Россия \\ ${ }^{2}$ Казанский национальный исследовательский технический университет им. А.Н. Туполева-КАИ, \\ 420111 Казань, Россия \\ e-mail: niazn@mail.ru
}

Поступило в Редакцию 28 марта 2019 г.

В окончательной редакции 28 марта 2019 г.

Принято к публикации 15 апреля 2019 г.

\begin{abstract}
Приведены результаты исследования изменения магнитной структуры планарных пермаллоевых микрочастиц под действием механического напряжения. Частицы были сформированы на стеклянных подложках и имели квадратную форму в плоскости образца. Было показано, что по изображениям таких частиц, полученным с помощью магнитно-силового микроскопа, можно детектировать одноосные механические напряжения. В зависимости от геометрических размеров частиц были определены диапазоны напряжений, для детектирования которых они могут эффективно использоваться.
\end{abstract}

Ключевые слова: планарные магнитные микрочастицы, магнитно-силовая микроскопия, магнитоупругий эффект.

DOI: $10.21883 / J T F .2019 .11 .48325 .113-19$

\section{Введение}

Магнитоупругий эффект (эффект Виллари) или влияние механических напряжений на магнитные свойства ферромагнитных материалов изучается и практически используется довольно долгое время. В последнее время основные усилия в данном направлении связаны с возможностью использования данного эффекта для управления намагниченностью микро- и наночастиц [1-5]. Это обусловлено тем, что использование магнитоупругого эффекта позволяет существенно снизить энергию, необходимую для перемагничивания однодоменной частицы, на базе которой могут быть созданы электронные логические элементы для обработки и хранения информации $[3,4]$. При этом для исследования намагниченности отдельно лежащей планарной частицы часто применяется магнитно-силовая микроскопия (МСМ) как один из наиболее перспективных методов, с помощью которого можно изучать распределение намагниченности в планарных микро и наноструктурах с пространственным разрешением до $30 \mathrm{~nm}$.

С другой стороны, на основе анализа МСМ изображения микрочастицы можно решить и обратную задачу получить данные о степени механического напряжения в частице и в случае одноосного воздействия определить его направление. При этом тонкие пленки [6-8] и планарные частицы [5] являются перспективными объектами для получения информации о распределении напряжения в приповерхностном слое подложки, на которой они расположены. При этом планарные частицы могут позволить детектировать механическое напряже- ние с разрешением, сопоставимым с их латеральными размерами (порядка $10 \mu \mathrm{m})$. Данное разрешение гораздо выше, чем разрешающая способность существующих тензодатчиков, разрешение которых также сопоставимо с их размерами от $1 \mathrm{~mm}$ и выше. Разрешением, сопоставимым с детектированием напряжений при помощи ферромагнитных микрочастиц, обладает спектроскопия комбинационного рассеяния света (рамановская спектроскопия) и одна из ее разновидностей - ближнеполевая рамановская спектроскопия [9-11]. Ближнеполевая спектроскопия позволяет добиться более высокого разрешения по сравнению с ферромагнитными микрочастицами, однако с ее помощью нельзя исследовать металлические поверхности. Ее обычно используют для проведения исследований на кремниевых структурах $[9,10]$ или многослойных структурах, созданных на их основе [11]. $\mathrm{У}$ планарных частиц тоже есть ограничения по исследуемым поверхностям: поверхность должна быть ровной и не взаимодействовать с магнитной структурой частиц.

В настоящей работе исследовалась возможность использования магнитоупругого эффекта для детектирования механических напряжений, возникающих в планарной пермаллоевой (Ру) микрочастице. Для этого изучалось изменение доменной структуры Ру микрочастицы в зависимости от внешних механических напряжений методами МСМ и компьютерного моделирования. На примере квадратных Ру-частиц было показано, что при механическом воздействии происходит изменение их магнитной структуры, которое может быть численно охарактеризовано и использовано для оценки механического напряжения в частице. Показано, что диапазон ме- 


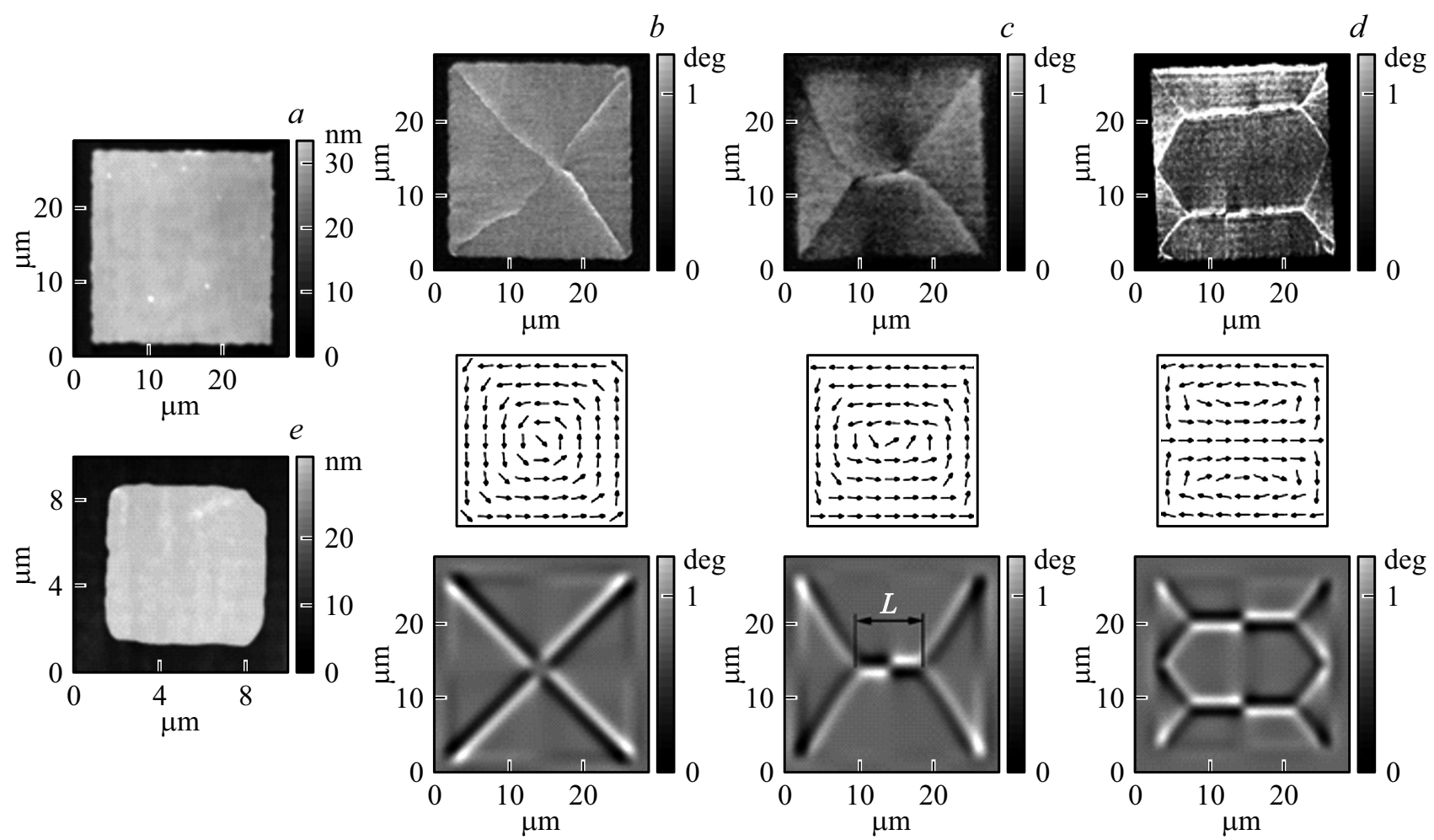

Рис. 1. Топографическое изображение Ру-частицы размерами $25 \times 25 \times 0.03 \mathrm{mkm}(a)$ и ее экспериментальные МСМ изображения: в 4-доменном состоянии при отсутствии механических напряжений $(b)$, в измененном 4-доменном состоянии под действием механических напряжений $(c)$, в 7-доменном состоянии под действием механических напряжений $(d)$. Под каждым изображением приведены соответствующие им схема распределения намагниченности в частице и модельное МСМ изображение. Буквой $L$ обозначена длина перемычки между доменами. Топографическое изображение Ру-частицы размерами $8 \times 8 \times 0.03 \mu \mathrm{m}(e)$.

ханических напряжений, который можно детектировать при помощи планарной частицы, зависит от ее размера.

\section{1. Методики подготовки исследуемых образцов и проведения МСМ измерений}

Исследования, проведенные в настоящей работе, выполнялись на частицах двух размеров. Это были планарные частицы квадратной формы с размерами $25 \times 25 \mu \mathrm{m}$ (рис. $1, a)$ и $8 \times 8 \mu \mathrm{m}$ (рис. $1, e)$ в плоскости образца и высотой, которая изменялась от образца к образцу в диапазоне от 10 до $50 \mathrm{~nm}$. Частицы были изготовлены из пермаллоя марки 79НМ (основной состав: $\mathrm{Ni} 79 \%$, $\mathrm{Fe} 16 \%$, Мо 4\%). Для создания образцов использовалось напыление через квадратную металлическую сетку, которое проводилось методом распыления твердотельной мишени электронным лучом в сверхвысоковакуумной установке Multiprobe P (Omicron). В качестве подложки для формирования частиц использовались стеклянные пластины размерами $14 \times 4 \mathrm{~mm}$ и толщиной $0.15 \mathrm{~mm}$. При этом сетка на поверхности подложки закреплялась таким образом, чтобы одна из сторон формируемой частицы была параллельна длинной стороне подложки.
Для 25-ти микронных частиц область с частицами занимала центр подложки и имела размер около $14 \times 4 \mathrm{~mm}$. При этом расстояние между сторонами сформированных частиц составляло примерно $8 \mu \mathrm{m}$. В случае 8-ми микронных частиц занимаемая ими область имела диаметр около $3 \mathrm{~mm}$ и находилась в центре подложки. Расстояние между сторонами частиц на данном образце составляло примерно $5.5 \mu \mathrm{m}$. Для обоих образцов расстояние между частицами было достаточным для того, чтобы частицы не оказывали влияния на магнитную структуру друг друга. Подложки с сформированными частицами отжигались в условиях сверхвысокого вакуума при температуре $300^{\circ} \mathrm{C}$ в течении $15 \mathrm{~min}$ для того, чтобы снять возможные механические напряжения, возникающие в частицах во время их напыления.

Для МСМ измерений полученных частиц использовались сканирующие зондовые микроскопы Solver P47 и Solver HV (НT-МДТ), оснащенные магнитными зондами NSC 18/Co-Cr (MikroScience) и Multi75M-G (BudgetSensors). Основные измерения частиц были выполнены по однопроходной методике, при которой МСМ зонд перемещается параллельно поверхности образца на достаточном удалении от нее (на расстоянии несколько десятков нанометров) для того, чтобы минимизировать влияние магнитного поля зонда на магнитную струк- 
туру частицы. Проведенные измерения показали, что получать стабильные магнитные изображения можно от частиц, высота которых равна или больше $20 \mathrm{~nm}$. На частицах высотой меныше $20 \mathrm{~nm}$ при сканировании часто наблюдалось изменение получаемого изображения под действием перемещающегося МСМ зонда.

Распределение намагниченности в частице по полученным МСМ изображениям определялось следующим образом. На основе изображения частицы, полученного в режиме атомно-силовой микроскопии, определялись ее размеры и по ним при помощи программы OOMMF [12] рассчитывалось возможное распределение намагниченности в частице. Затем по полученному распределению создавалось виртуальное МСМ изображение при помощи разработанной ранее программы „Виртуальный микроскоп“ [13]. Виртуальное изображение сравнивалось с экспериментальным и при их совпадении делался вывод о том, что полученное в OOMMF распределение намагниченности соответствует реальному.

Для создания механических напряжений в частицах использовался специальный держатель образца, устанавливаемый в зондовый микроскоп. Он представлял собой ровную металлическую немагнитную пластину, при этом под центр образца подкладывалась вольфрамовая проволока перпендикулярно длинной стороне подложки, а ее концы прижимались к поверхности держателя специальными зажимами. Согласно проведенным расчетам, это позволяет создать в центре подложки одноосные растягивающие напряжения, направленные перпендикулярно оси подложенной проволоки, значение которых изменяется от центра к краю образца. При этом направление растяжения частицы оказывается параллельным одной из ее сторон, что важно с точки зрения интерпретации полученных результатов. За счет изменения толщины подкладываемой проволоки можно было изменять диапазон механических напряжений в частицах.

\section{2. Влияние механических напряжений на магнитную структуру пермаллоевой частицы}

Согласно полученным МСМ изображениям (рис. 1), ненапряженная Ру-частица имеет классическую четырехдоменную (4-доменную) структуру (рис. $1, b)$ с доменами одинаковыми по размерам. У напряженных Ру-частиц наблюдается перераспределение локальных магнитных моментов (рис. 1,c). Происходит увеличение размера доменов с намагниченностью, направленной перпендикулярно оси растяжения (рис. 1,c). Это обусловлено отрицательной константой магнитострикции используемого материала. За счет увеличения площади этих двух доменов в центре частицы между ними образуется доменная стенка, наблюдаемая на МСМ изображении, как характерная перемычка (рис. 1, c). Для 25-ти микронных частиц при больших значениях механического напряжения кроме 4-доменного состояния также наблюдалось и семидоменное (7-доменное) состояние (рис. 1, $d$ ). В 7-доменном состоянии у частицы образуются две приблизительно равные по размеру перемычки между доменами с направлением намагниченности, перпендикулярным направлению растяжения. Длина образовавшейся перемычки может быть легко определена по MCM изображению частицы и также, как и для частиц в 4-доменном состоянии, в дальнейшем использована для определения значения механического напряжения, воздействующего на частицу.

Как известно, плотность энергии анизотропии зависит от механического напряжения как

$$
E_{a}=-1.5 \cdot \lambda \cdot \sigma\left(\cos ^{2} \alpha-1 / 3\right),
$$

где $\lambda$ - константа магнитострикции, $\sigma$ - напряжение, $\alpha$ - угол между направлением намагниченности и приложенного одноосного напряжения [14]. Поэтому, для того чтобы сопоставить длину перемычки с механическим напряжением, с помощью OOMMF была рассчитана зависимость длины перемычки от плотности энергии анизотропии, наведенной этим напряжением. При проведении расчетов в программе ООММF варьируется ориентация локальных магнитных моментов для каждой ячейки, на которые разбивается общий объем частицы с тем, чтобы минимизировать ее полную энергию. При этом учитывается обменное взаимодействие, магнитостатическая энергия, энергия анизотропии частицы и энергия частицы во внешнем магнитном поле (в нашем случае данная энергия не учитывалась, так как внешнее поле отсутствует). Для проведения расчетов необходимо задавать предполагаемое распределение намагниченности, так как программа минимизирует энергию для начального распределения магнитных моментов и не может корректно рассчитать переход из одного состояния в другое. Поэтому в нашем случае при моделировании для частиц с заданными размерами варьировалось два параметра: константа анизотропии и форма начального распределения намагниченности.

По полученным результатам были построены зависимости плотности полной энергии частицы от плотности энергии анизотропии для всех размеров исследуемых частиц. Анализ полученных зависимостей показал, что для 8-ми микронных частиц энергетически выгодным оказывается 4-доменное состояние при любых значениях механического напряжения (рис. 2, $a$ ). Для 25-ти микронных частиц 4-доменное состояние выгодно только при низких значениях механического напряжения (рис. 2,b). После определенного значения $E_{a}$ полная энергия частицы начинает резко возрастать при небольшом изменении $E_{a}$ и пересекает график с зависимостью энергии для частицы в 7-доменном состоянии. В окрестностях этой точки возможно существование как 4-доменного, так и 7-доменного состояния частицы. При дальнейшем увеличении $E_{a}$ более выгодным становиться 7-доменное 

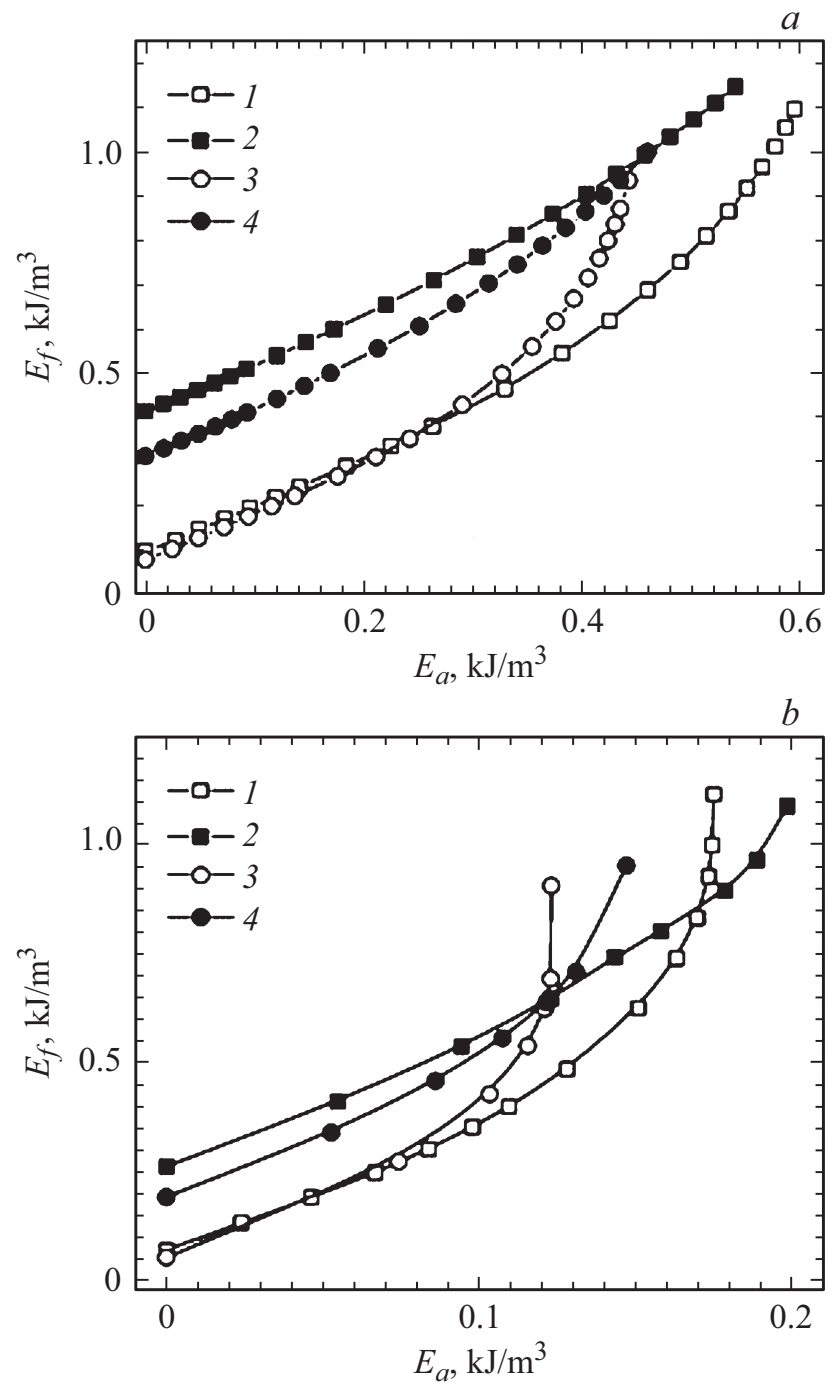

Рис. 2. Рассчитанная зависимость плотности полной энергии $\left(E_{f}\right)$ от плотности энергии анизотропии $\left(E_{a}\right)$ для 8-микронных (a) и 25-микронных $(b)$ частиц. Приведены результаты расчета для частиц высотой $30 \mathrm{~nm}$ в 4-доменном (обозначено цифрой 1) и 7-доменном (2) состояниях и высотой $20 \mathrm{~nm}$ в 4-доменном (3) и 7-доменном (4) состояниях.

состояние, так как в таком состоянии полная энергия частицы оказывается меньше. Данный переход происходит при меньших значениях действующего механического напряжения с уменьшением высоты частицы (рис. 2,b). Резкое увеличение $E_{a}$ также свидетельствует о том, что обсчитываемая 4-доменная модель частицы перестает работать корректно.

Как показало моделирование, длина перемычки, образующейся между доменами, также зависит от высоты частицы (рис. 3,a). Таким образом, чем меньше высота частицы, тем более чувствительной оказывается ее магнитная структура к механическим напряжениям. Поэтому лучшей для визуализации механических напряжений при помощи МСМ измерений оказывается частица с минимальной высотой, которой достаточно для того, чтобы при сканировании МСМ зондом не происходило искажения структуры намагниченности этой частицы. В нашем случае стабильные изображения получались на частицах высотой $20 \mathrm{~nm}$ и более. Возможно данное значение может быть снижено при использовании МСМ зондов с низким магнитным моментом. Исходя из формулы (1), полученные зависимости длины перемычки от энергии анизотропии можно пересчитать в зависимость длины перемычки от значения механического напряжения и подбирать частицы нужных размеров для определения этих напряжений на основе МСМ изображения частицы (рис. 3,b). При расчетах константа магнитострикции принималась равной $-3 \cdot 10^{-6}$. Исходя из полученных данных, можно сказать, что увеличение латеральных размеров частицы приводит к уменьшению значения механического напряжения, необходимого для изменения ее магнитной структуры, и снижает диапазон механических
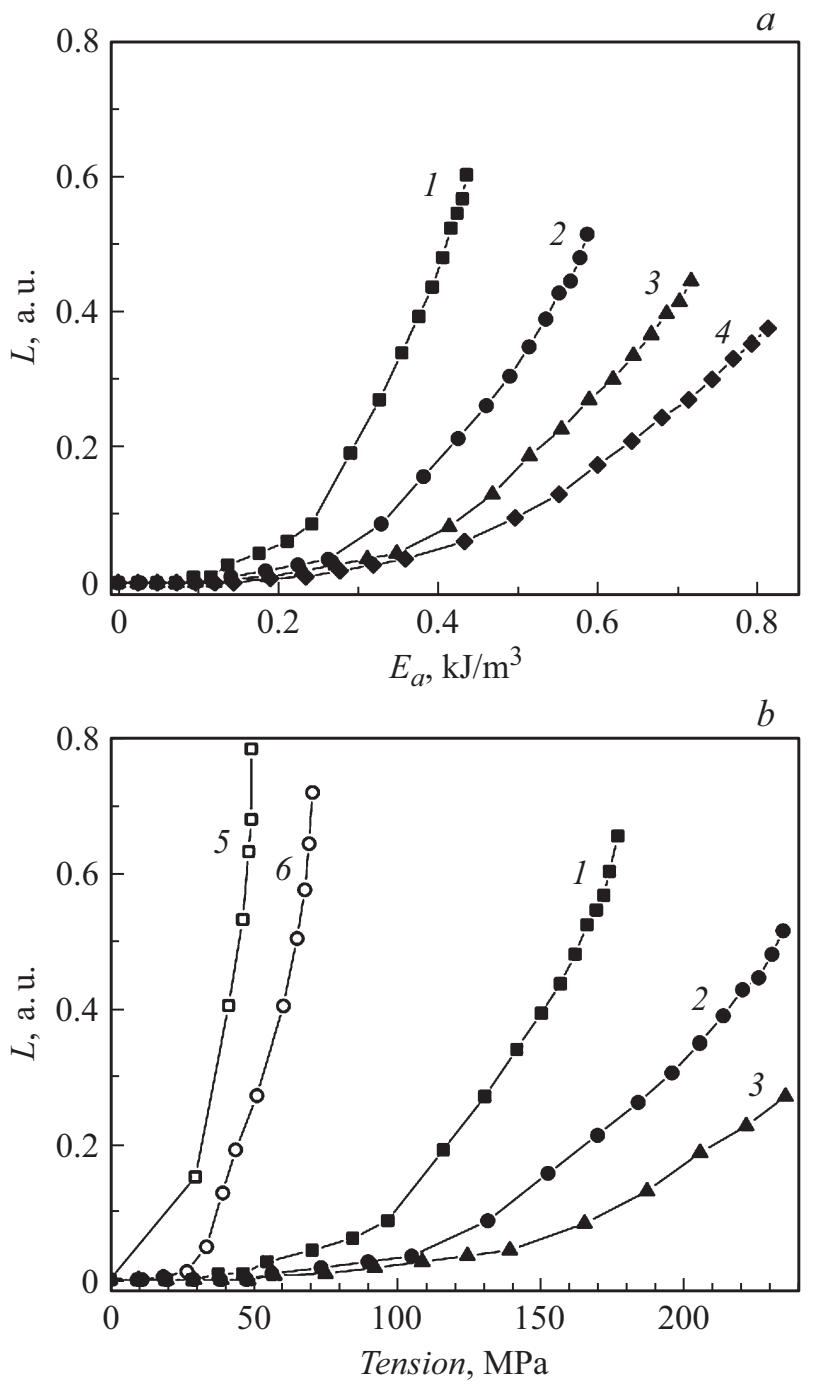

Рис. 3. Рассчитанная зависимость относительной длины $(L)$ перемычки между доменами от плотности энергии анизотропии $(a)$ и механического напряжения $(b)$ для 8-микронных частиц высотой 20 (обозначено цифрой 1), 30 (2), 40 (3), $50 \mathrm{~nm}(4)$ и 25 -микронных частиц высотой 20 (5), $30 \mathrm{~nm}(6)$. 

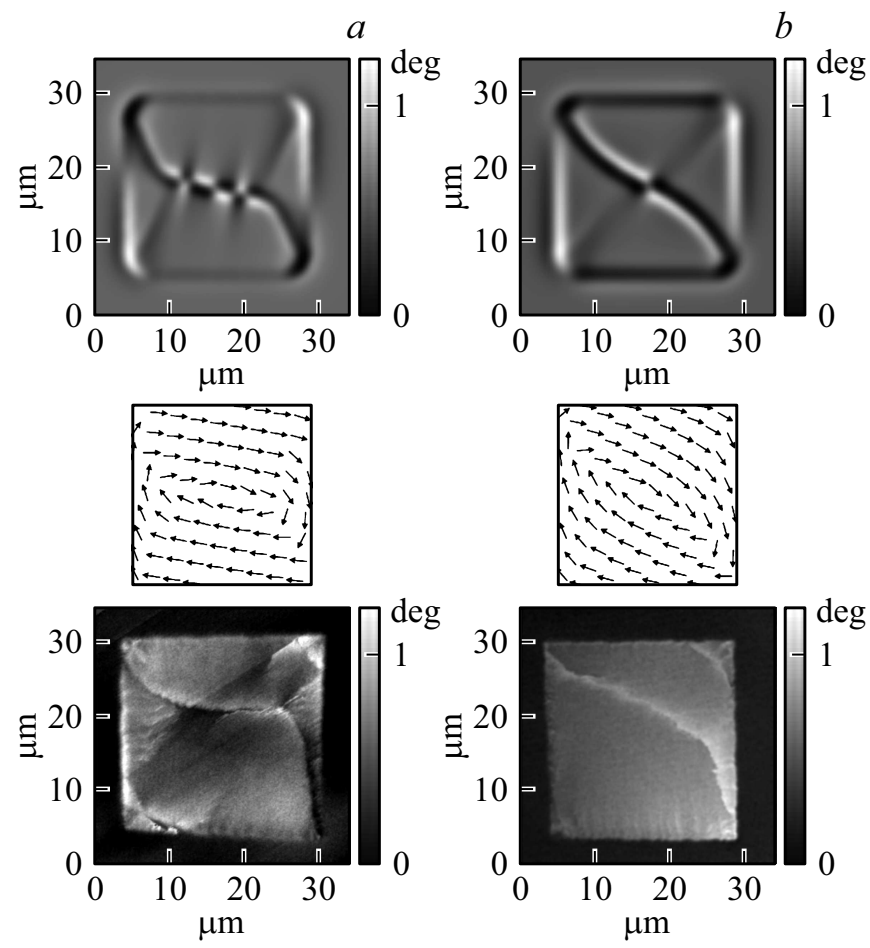

Pис. 4. Изображения 25-микронной частицы при условии, что частица растягивается в направлении, которое имеет угол с ее стороной равный $10^{\circ}(a)$ и $30^{\circ}(b)$. Последовательно сверху вниз представлены: виртуальное МСМ изображение, схема распределения намагниченности, смоделированная при помощи OOMMF, и экспериментальное МСM изображение.

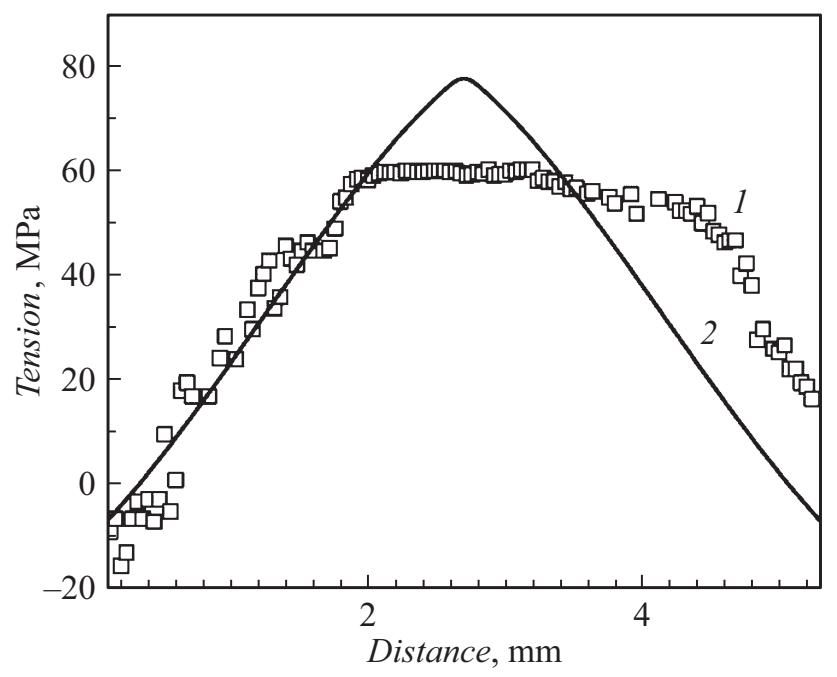

Рис. 5. Распределение напряжения в неравномерно изогнутом образце в зависимости от расстояния, полученное на основе анализа МСМ изображений частиц (1) и расчет (2).

напряжений, в котором происходит изменение длины перемычки.

При помощи моделирования также была проведена оценка изменений в распределении намагниченности при условии, что направление механического напря- жения, действующего на частицу, имеет определенный угол с ее стороной. Оценивалась возможность корректного определения длины перемычки между доменами и нахождения, исходя из нее, значения действующего механического напряжения. Было установлено, что для таких частиц 4-доменная структура, с перемычкой между двумя увеличенными доменами, сохраняется до тех пор, пока угол между направлением одноосного напряжения и стороной частицы составляет менее $25^{\circ}$ (рис. 4,a). При больших углах частицы становятся 2-доменными и по их МСМ изображению можно сделать вывод только о наличии механического напряжения и нельзя оценить его значение (рис. 4, $b$ ). Экспериментальные МСМ изображения, полученные на 25-микронных частицах, подтверждают результаты моделирования (рис. 4).

Для экспериментальной проверки выводов о возможности использования перемычки между доменами в качестве детектора механического напряжения был взят образец с 25-миконными частицами высотой $30 \mathrm{~nm}$. Образец был закреплен в специальном держателе. Под центр образца была подложена вольфрамовая проволока диаметром $50 \mu \mathrm{m}$, а его края прижаты к ровному основанию с помощью специальных зажимов. При этом свободной осталась область длиной около $5 \mathrm{~mm}$ в центре образца, в которой был создан градиент механических напряжений. Значения механических напряжений вдоль центральной линии образца были рассчитаны методом конечных элементов (рис. 5) [15]. При этом часть образца около зажимов испытывало одноосное сжатие, которое отражено на графике в виде отрицательных значений, а центральная часть - растяжение. Затем образец был последовательно просканирован вдоль центральной оси с помощью МСМ и, исходя из полученных длин перемычек, была построена экспериментальная зависимость значений механического напряжения вдоль этой оси (рис. 5). В центре образца (диапазон 2-4 mm) наблюдается практически постоянное значение механического напряжения, что связано с тем, что, согласно рис. $3, b$, изменения длины перемычки в диапазоне от 0.6 до 1 практически не изменяют энергию анизотропии (и соответствующее ей механическое напряжение). Поэтому, какая бы перемычка не наблюдалась в этом диапазоне, механическое напряжение, рассчитанное, исходя из ее длины, будет практически постоянным. Возможно, при большом увеличении значения механического напряжения произойдет переход частицы из многодоменного в однодоменное состояние. Частицы в однодоменном состоянии наблюдались нами на МСМ изображениях, однако корректно оценить величину механического напряжения, необходимого для перехода из одного состояния в другое, не представляется возможным. Небольшое расхождение между расчетным и экспериментальным распределением механических напряжений в области после $4 \mathrm{~mm}$, по всей видимости, может быть связано с недостаточным прижатием одного из краев образца при эксперименте. Полученные результаты показывают, что по изменению распределения намагниченности пла- 
нарной ферромагнитной частицы можно детектировать одноосные механические напряжения.

\section{Заключение}

На примере планарных пермаллоевых частиц квадратной формы было показано, что по изменению магнитной структуры частицы можно численно оценить наведенное в ней одноосное механическое напряжение. В случае квадратных в плоскости частиц (при длине стороны от 5 до $30 \mu \mathrm{m})$ в отсутствие механического напряжения ее магнитная структура является четырехдоменной, с доменами, равными по размерам. Под действием механического напряжения размер двух доменов увеличивается и между ними образуется перемычка, длина которой пропорциональна приложенному напряжению. При этом за счет подбора геометрических размеров частиц можно выбирать диапазон механических напряжений, в котором происходит изменение распределения намагниченности в частице, которое можно численно охарактеризовать по МСМ изображению. В частности, было показано, что частицы размерами $25 \times 25 \mu \mathrm{m}$ эффективны для детектирования механических напряжений в диапазоне 20-80 MPa, а частицы $8 \times 8 \mu \mathrm{m}$ в диапазоне 120-280 MPa.

\section{Финансирование работы}

Работа выполнена при поддержке Российского фонда фундаментальных исследований (грант № 17-08-00915).

\section{Конфликт интересов}

Авторы заявляют, что у них нет конфликта интересов.

\section{Список литературы}

[1] Морозов А.И. // ФТТ. 2014. Т. 56. Вып. 5. С. 833-840. [Morosov A.I. // Phys. Solid State. 2014. Vol. 56. N 5. P. 865872. DOI: https://doi.org/10.1134/S1063783414050199]

[2] Bur A., Wu T., Hockel J., Hsu C., Kim H., Chung T., Wong K., Wang K., Carma G. // J. Appl. Phys. 2011. Vol. 109. P. 123903. DOI: https://doi.org/10.1063/1.3592344

[3] Nikonov D.E., Young I.A. // J. Mater. Res. 2014. Vol. 29. N 18. P. 2109-2115. DOI: https://doi.org/10.1557/jmr.2014.243

[4] Бухараев А.А., Звездин А.К., Пятаков А.П., Фетисов Ю.К. // УФН. 2018. Т. 188. № 12. С. 1288 1330. DOI: 10.3367/UFNr.2018.01.038279 [Bukharaev A.A., Zvezdin A.K., Pyatakov A.P., Fetisov Yu.K. // Phys. Usp. 2018. Vol. 61. P. 1175-1212. DOI: 10.3367/UFNe.2018.01.038279]

[5] Finizio S., Foerster M., Buzzi M., Kruger B., Jourdan M., Vaz C., Hockel J., Miyawaki T., Tkach A., Valencia S., Kronast F., Carman G., Nolting F., Klaui M. // Phys. Rev. Appl. 2014. Vol. 1. P. 021001.

DOI: 10.1103/PhysRevApplied.1.021001

[6] Dai G., Zhan Q., Yang H., Liu Y., Zhang X., Zuo Z., Chen B., Li R. // J. Appl. Phys. 2013. Vol. 114. P. 173913. DOI: http://dx.doi.org/10.1063/1.4829670
[7] Беляев Б.А., Изотов А.В. // ФТТ. 2007. Т. 49. Вып. 9. C. 1651-1659. [Belyaev B.A., Izotov A.V. // Phys. Solid State. 2007. Vol. 49. N 9. P. 1731-1739. https://doi.org/10.1134/S106378340709020X]

[8] Cao D., Wang Z., Pan L. // Appl. Phys. A. 2016. Vol. 122. P. 938. DOI: 10.1007/s00339-016-0468-y

[9] Wolf I. // Semicond. Sci. Technol. 1996. Vol. 11. P. 139-154.

[10] Srikaret V., Swan A., Unlu M., Goldberg B., Spearing S. // J-MEMS. 2003. Vol. 12. N 6. P. 779-787.

[11] Satohet A., Tada T., Poborchiial V., Kanayama T., Satoh S., Arimoto H. // Jpn. J. Appl. Phys. 2012. Vol. 51. P. 016603. DOI: 10.1143/JJAP.51.016603

[12] Donahue M.J., Porter D.G. OOMMF. http://math.nist.gov/oommf/

[13] Овчинников Д.В., Бухараев А.А. // ЖТФ. 2001. Т. 71. Вып. 8. C. 85-91. [Ovchinnikov D.V., Bukharaev A.A. // Tech. Phys. 2001 Vol. 46. N 8. P. 1014-1019. DOI: https://doi.org/10.1134/1.1395123]

[14] Боровик Е.С., Еременко В.В., Мильнер А.С. Лекции по магнетизму. М.: Физматлит, 2005. 512 с.

[15] NX Nastran. Theoretical Manual. // Siemens Product Lifecycle Management Software Inc. 2008. 